\title{
Theory of reduced superfluid density in underdoped cuprate superconductors
}

\author{
Wei-Cheng Lee, ${ }^{1}$ * Jairo Sinova, ${ }^{2}$ A. A. Burkov, ${ }^{3}$ Yogesh Joglekar, ${ }^{4}$ and A. H. MacDonald ${ }^{1}$ \\ ${ }^{1}$ Department of Physics, The University of Texas at Austin, Austin, Texas 78712, USA \\ ${ }^{2}$ Department of Physics, Texas A\&M University, College Station, Texas 77843-4242, USA \\ ${ }^{3}$ Department of Physics and Astronomy, University of Waterloo, Waterloo, Ontario, Canada N2L 3G1 \\ ${ }^{4}$ Department of Physics, Indiana University-Purdue University Indianapolis, Indianapolis, Indiana 46202, USA
}

(Received 6 May 2008; published 19 June 2008)

\begin{abstract}
The critical temperature of an underdoped cuprate superconductor is limited by its phase stiffness $\rho$. In this paper we argue that the dependence of $\rho$ on doping $x$ should be understood as a consequence of deleterious competition with antiferromagnetism at large electron densities, rather than as evidence for pairing of holes in the $x=0$ Mott insulator state. $\rho$ is suppressed at small $x$ because the correlation energy of a $d$-wave superconductor has a significant pairing-wave-vector dependence when antiferromagnetic fluctuations are strong.
\end{abstract}

DOI: 10.1103/PhysRevB.77.214518

\section{INTRODUCTION}

The fascinating and rich phenomenology of hightemperature cuprate superconductors has been very thoroughly studied over the past 20 years. Although there is substantial variability in details from material to material, all cuprates exhibit robust Mott insulator antiferromagnetism when the hole-doping fraction $x$ is very small, superconductivity which appears when $x$ exceeds a minimum value $\sim 0.1$, and a maximum $T_{c}$ in optimally doped materials with $x$ $\sim 0.2$. In the underdoped regime, the superconducting transition temperature is limited by phase fluctuations, ${ }^{1-4}$ and experiments hint at a wide variety of (typically) short-range correlations associated with competing charge and spin orders. The underdoped regime poses a fundamental challenge to theory because its electronic properties are not fully consistent with any of the various well-understood fixed-point behaviors that often help us to classify and predict the properties of very complex materials.

The phenomenological parameter $\rho$ used to characterize phase-fluctuation stiffness in a superconductor is normally expressed in terms of the superfluid density $n_{s}$ by writing $\rho$ $=\hbar^{2} n_{s} / m^{*}$, an identification that is partly justified by BCS mean-field theory. The increase in $\rho$ with $x$ in cuprate superconductors is therefore readily accounted for by theories ${ }^{5}$ in which superconductivity is due to the condensation of Cooper pairs formed from holes in a doped Mott insulator. ${ }^{6}$ Theories which start with this view must still explain the fact that $\rho$ vanishes at a nonzero value of $x$, and deal with the awkward property that cuprate superconductivity evolves smoothly from the underdoped regime to an overdoped regime, which appears to be explainable in terms of conventional band-quasiparticle Cooper pair condensation. In this paper we propose an alternate explanation for the $x$ dependence of $\rho$ based on band-quasiparticle pairing. Our argument accounts for the correlation energy of a $d$-wave superconductor in the presence of incipient antiferromagnetism and is based on the following general expression for the phase stiffness of a superconductor:

$$
\rho=\frac{1}{A} \frac{d^{2} E}{d P^{2}},
$$

where $A$ is the area of the system, $\vec{P}$ is the pairing wave vector, $^{7}$ and $E$ is the total energy including both mean-field
PACS number(s): 74.20.-z, 74.72.-h, 71.27.+a

and correlation contributions, $E=E^{\mathrm{MF}}+E^{\mathrm{cor}}$. The familiar BCS theory expression for $\rho$ captures only the mean-field theory contribution to the energy.

When superconductivity is viewed as a weak-coupling instability of a Fermi liquid, it is usually implicitly assumed that $E^{\text {cor }}$ is not significantly influenced by the formation of the superconducting condensate, and certainly not by changes in the condensate's pairing momentum $\vec{P}$. In the case of simple models with parabolic bands and Galilean invariance, neglect of the correlation energy contribution can be justified rigorously. We argue the correlation energy contribution is significant in underdoped cuprates because there is direct competition between the Fermi sea quantum fluctuations which condense in antiferromagnetic and $d$-wave superconducting states. Consequently the pair-breaking effects of finite $\vec{P}$, which weaken superconductivity, also increase the importance of antiferromagnetic fluctuations, lowering $E^{\text {cor }}$ and decreasing $\rho$ compared to its mean-field value. In Secs. II and III, we first use a fully phenomenological and then a partially microscopic extended-Hubbard-model weakcoupling theory to expand on this idea. The conjugate relationship 8 between pairing and antiferromagnetic fluctuations plays an important role in the fluctuation spectrum and hence in theories of the correlation energy. In our theory of the underdoped state, the resonant magnetic mode observed in inelastic neutron scattering ${ }^{9,10}$ experiments, known as inelastic neutron scattering resonance (INSR), therefore has a somewhat different interpretation from that in most earlier theories, ${ }^{11-14}$ appearing as a kind of magnetic plasmon.

\section{PHENOMENOLOGICAL THEORY}

The basic ideas of our theory are qualitative, independent of most microscopic details, and most easily described in terms of the properties of a low-energy effective-field model for the collective fluctuations of a weak-coupling $d$-wave superconductor. The relationship to less transparent generalized random-phase approximation (GRPA) correlation energy calculations is explained below. We construct a quantum action by introducing a set of states which incorporate the coupled triplet-pairing and spin-density fluctuations, on which we focus. $|\Psi[\phi, V]\rangle$ is the Fock-space Slater determinant ground state of the quadratic Hamiltonian 


$$
\begin{aligned}
\mathcal{H}^{\text {fluc }}= & \mathcal{H}_{\mathrm{MF}}+\sum_{i \sigma} \sigma V_{i} c_{i \sigma}^{\dagger} c_{i \sigma}+\Delta_{0}\left\{\sum _ { i \tau } ( - ) ^ { \tau } \left[\exp \left(i \phi_{i}\right)\right.\right. \\
& \left.-1] c_{i \uparrow}^{\dagger} c_{i+\tau \downarrow}^{\dagger}+\text { H.c. }\right\} .
\end{aligned}
$$

(For notational simplicity we have exhibited here only fluctuations with zero spin projection along the quantization direction.) In Eq. (2), $\tau$ labels the four neighbors of each site on a two-dimensional square lattice, and $(-)^{\tau}$ represents the $d$-wave variation of mean-field near-neighbor pair potentials. Using these states as an approximate identity resolution leads to the following low-energy imaginary-time action for the collective variables $\phi_{i}$ and $V_{i}$ :

$$
\mathcal{S}=\int_{0}^{\infty} d \tau\left(\hbar\left\langle\Psi[\phi, V]\left|\partial_{\tau}\right| \Psi[\phi, V]\right\rangle+E[\phi, V]\right),
$$

where $E[\phi, V]=\langle\Psi[\phi, V]|\mathcal{H}| \Psi[\phi, V]\rangle$ and $\mathcal{H}$ is the full microscopic Hamiltonian. Mean-field-theory states are obtained by minimizing $E[\phi, V]$. The first term in the action captures the Berry phase coupling ${ }^{8}$ between pairing and spin-density fluctuations which we now elaborate.

The potentials associated with the two types of fluctuations are

$$
\begin{gathered}
\partial \mathcal{H}^{(\mathrm{fluc})} / \partial V_{\vec{k}}=\sum_{\sigma, \vec{p}} \sigma c_{\vec{p}-\vec{k}, \sigma}^{\dagger} c_{\vec{p}, \sigma} \\
\partial \mathcal{H}^{(\mathrm{fluc})} / \partial \phi_{\vec{k}}=i \sum_{\vec{p}} \Delta_{\vec{p}}\left[c_{\vec{p}-\vec{k}, \uparrow}^{\dagger} c_{-p, \downarrow}^{\dagger}-\text { H.c. }\right] .
\end{gathered}
$$

The Berry phase term can be evaluated explicitly for small fluctuations by using perturbation-theory expressions for the wave functions which appear in the Slater determinant $|\Psi[\phi, V]\rangle$ as follows:

$$
\mathcal{S}_{\text {Berry }}=\int_{0}^{\infty} d \tau \sum_{\vec{k}} C_{\vec{k}} \phi_{-\vec{k}} \partial_{\tau} V_{\vec{k}},
$$

where

$$
C_{\vec{k}}=2 \sum_{\vec{p}} \frac{\operatorname{Im}\left[\left\langle\chi_{\vec{p},-}\left|\frac{\partial \mathcal{H}^{\text {fluc }}}{\partial V_{\vec{k}}}\right| \chi_{\vec{p}+\vec{k},+}\right\rangle\left\langle\chi_{\vec{p}+\vec{k},+}\left|\frac{\partial \mathcal{H}^{\text {fluc }}}{\partial \phi_{-\vec{k}}}\right| \chi_{\vec{p},-}\right\rangle\right]}{\left(E_{\vec{p}+\vec{k}}+E_{\vec{p}}\right)^{2}} .
$$

In Eq. (6) we have made the usual Nambu spin-down particle-hole transformation of the mean-field Hamiltonian so that it has two eigenstates at each wave vector in the square lattice Brillouin zone with eigenvalues $\pm E_{\vec{p}}$, one $\left(\chi_{\vec{p},-}\right)$ occupied and one $\left(\chi_{\vec{p},+}\right)$ unoccupied. In Fig. 1 we show Berry curvature values calculated from this expression as a function of $\vec{k}$ which are strongly peaked near $\vec{k}=\vec{Q}$ $=(\pi / a, \pi / a)$; these results are robust over a broad range of dopings, gap sizes, and band-structure models. Pairing phase fluctuations are conjugated to spin-density fluctuations for $\vec{k}$ near $\vec{Q}$, just as they are conjugated to charge-density fluctuations for $\vec{k}$ near 0 , because of ${ }^{8}$ the $d$-wave property $\Delta_{\vec{p}+Q}=$ $-\Delta_{\vec{p}}$.

We now argue that there is competition between the correlation energy gain due to antiferromagnetic fluctuations and $d$-wave singlet Cooper pair formation. Strong experimental evidence for this competition is provided by the ap-

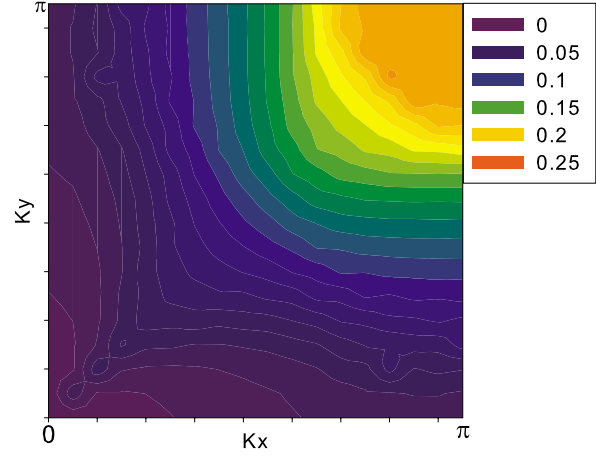

FIG. 1. (Color online) Berry's curvature $C_{\vec{k}}$ vs $\vec{k}$ for the $d$-wave mean-field state of a generalized Hubbard model with $U / t=2.0$, $V / t=2.0, t^{\prime} / t=-0.3$, and $x=0.12 . \Delta=\left\langle c_{i \uparrow} c_{j \downarrow}\right\rangle=(-)^{\tau} 0.145$ in this case.

parent enhancement $t^{5,15}$ of antiferromagnetic fluctuations in cuprate vortex cores and in cuprates placed in an external magnetic field. Changes in external conditions which weaken superconductivity enhance antiferromagnetism. Here we explore the consequences of this competition for the correlation contribution to the superfluid density, i.e., for the dependence of correlation energy on pairing momentum $\vec{P}$.

In our model the quadratic fluctuation action of a $d$-wave superconductor is

$$
\begin{aligned}
\mathcal{L}_{\text {fluc }}= & \frac{1}{2 \beta} \sum_{\omega, \vec{k}}\left[-2 \hbar \omega C_{\vec{k}, \omega} V(-\vec{k},-\omega) \phi(\vec{k}, \omega)\right. \\
& \left.+K_{\vec{k}, \omega}^{\mathrm{sp}}|V(\vec{k}, \omega)|^{2}+K_{\vec{k}, \omega}^{\phi}|\phi(\vec{k}, \omega)|^{2}\right] .
\end{aligned}
$$

In Eq. (7), $K^{\phi}$ and $K^{\text {sp }}$ are phase and spin-density stiffness. The onset of antiferromagnetism occurs when $K_{Q, \omega=0}^{\mathrm{sp}}=0$. In using this action we assume that the most important quantum fluctuations are $d$-wave pair phase and spin fluctuations. The microscopic GRPA calculations described in Sec. III support this assumption. In Eq. (7), frequency dependence is indicated in $C_{\vec{k}}, K_{\vec{k}}^{\mathrm{sp}}$, and $K_{\vec{k}}^{\phi}$ to recognize the existence of nonadiabatic effects accounted for in these more microscopic calculations but neglected in this qualitative discussion. The quadratic fluctuation action then describes a system with collective modes at energies

$$
E_{\vec{k}}^{\mathrm{res}}=\frac{\sqrt{K_{\vec{k}}^{\mathrm{sp}} K_{\vec{k}}^{\phi}}}{C_{\vec{k}}},
$$

and a corresponding zero-point energy contribution

$$
E^{\mathrm{zp}}=\sum_{k}^{\prime} E_{\vec{k}}^{\mathrm{res}} / 2 .
$$

This adiabatic theory of the INSR mode is accurate only when $E_{\vec{k}}^{\text {res }}$ lies below the particle-hole continuum; the prime on the wave-vector sum above recognizes that this condition is satisfied only near $\vec{k}=\vec{Q}$. The fluctuation correction to $\rho$ can be related to the pairing-wave-vector dependence of the zero-point energy as we explain below. 
We expect $K^{\mathrm{sp}}(\vec{Q})$ to decrease with $\vec{P}$ because suppressed pairing favors antiferromagnetism. The strength of this dependence can be estimated roughly from experiment by associating the magnetic length $\ell_{B}$ at the magnetic field strength required to induce antiferromagnetism in a cuprate superconductor with the value of $P^{-1}$ at which $K^{\mathrm{sp}}(\vec{Q})$ goes to zero. Taking a typical value for this field, $\sim 100 \mathrm{~T}$, and assuming that the resonance mode is well defined over the portion of the Brillouin zone (BZ) with large Berry curvature (say $\sim 10 \%$ ) gives a negative correlation energy contribution to the phase stiffness per two-dimensional cuprate layer of $\rho^{\text {cor }} \sim-0.1 n \ell_{B}^{2} E^{\text {res }} \sim-E^{\text {res }} \sim 0.05 \mathrm{eV}, \quad$ comparable to the value of $\rho$ inferred from penetration depth measurements in optimally doped samples. Although this estimate is clearly very rough, it does establish that the correlation correction to $\rho$ can be substantial in the underdoped regime.

Charge-density fluctuations are not included in this analysis because their Berry phase coupling to the phase fluctuations of the superconducting order parameter is large only near $\vec{k}=0$ and negligible near $\vec{k}=\vec{Q} .{ }^{16}$ Therefore the chargedensity fluctuations do not play a significant role in the physics near $\vec{k}=\vec{Q}$; instead they cause the instabilities near $\vec{k}=0$ in the microscopic GRPA calculations which we mention below.

\section{MICROSCOPIC GRPA THEORY}

We now evaluate the correlation energy of an extended Hubbard mode ${ }^{17}$ in the GRPA. The model we study in this paper has on-site repulsive interactions $U$, which drive antiferromagnetism, and near-neighbor attractive interactions $V$, which drive $d$-wave superconductivity: $H=H_{t}+H_{U}+H_{V}$, where

$$
\begin{gathered}
H_{t}=-t \sum_{\langle i, j\rangle, \sigma} c_{i \sigma}^{\dagger} c_{j \sigma}+\text { H.c. }-t^{\prime} \sum_{\langle i, j\rangle^{\prime}, \sigma} c_{i \sigma}^{\dagger} c_{j \sigma}+\text { H.c., } \\
H_{U}=U \sum_{i} \hat{n}_{i \uparrow} \hat{n}_{i \downarrow}, \quad H_{V}=-V \sum_{\langle i, j\rangle \sigma \sigma^{\prime}} \hat{n}_{i \sigma} \hat{n}_{j \sigma^{\prime}} .
\end{gathered}
$$

In Eq. (10) $U, V, t$, and $t^{\prime}$ should all be thought of as effective parameters which apply at the energy scale of pairing and depend on $x$. Values for $V, t$, and $t^{\prime}$ can be estimated from angle-resolved photoelectron spectroscopy (ARPES) data. ${ }^{18}$ Spin-dependent Heisenberg near-neighbor interactions of the type used in $t-J$ models could also be used in the low-energy effective Hamiltonian, but are neglected here for simplicity. The conclusions we draw in this paper do not depend on whether the near-neighbor effective interaction which drives $d$-wave superconductivity is spin independent or spin dependent.

The GRPA correlation energy of a $d$-wave condensate state with pairing momentum $\vec{P}$ is ${ }^{19}$

$$
E^{\operatorname{cor}}(\vec{P})=\frac{1}{2} \sum_{\vec{q}, i}\left[\omega_{i}(\vec{P}, \vec{q})-\epsilon_{i}^{\mathrm{ph}}(\vec{P}, \vec{q})\right],
$$

where $\epsilon_{i}^{\mathrm{ph}}(\vec{P}, \vec{q})$ is a quasiparticle particle-hole excitation energy, and $\omega_{i}(\vec{P}, \vec{q})$ is the corresponding GRPA excitation en- ergy. This equation can be derived by expanding the GRPA Hamiltonian to quadratic order in particle-hole excitation amplitudes approximated as independent bosons. The correlation energy expression then drops out of a boson Bogoliubov transformation. The analysis in the preceding qualitative discussion assumed that the $\vec{P}$ dependence of $E^{\text {cor }}$ is dominated by its collective-mode contribution, an assumption that is largely justified by the following microscopic calculations.

In a GRPA theory, excitation energies $\omega_{i}(\vec{P}, \vec{q})$ are obtained from a time-dependent mean-field theory ${ }^{19}$ in which the quasiparticles respond to the external potential and to induced mean-field potentials: $H^{\prime}=H^{\mathrm{ext}}+H^{f}(t)$, where $H^{f}(t)$ $=H^{f 1}(t)+H^{f 2}(t)$, with

$$
\begin{aligned}
& \left.H^{f 1}(t)=\frac{1}{A_{\vec{p}, \vec{k}, \vec{q}, \sigma}} \sum_{(\vec{q})}\left[\delta\left\langle c_{\vec{p}+\vec{q} \bar{\sigma}}^{\dagger} c_{\vec{p}}\right\rangle\right\rangle c_{\vec{k}-\vec{q} \sigma}^{\dagger} c_{\vec{k} \sigma}\right]+G(\vec{k}, \vec{p}, \vec{q}) \\
& \times\left[\delta\left\langle c_{\vec{p}+\vec{q} \sigma}^{\dagger} c_{\vec{p} \sigma}\right\rangle c_{\vec{k}-\vec{q} \sigma}^{\dagger} c_{\vec{k} \sigma}\right]+H(\vec{k}, \vec{p}) \\
& \times\left[\delta\left\langle c_{\vec{q}-\vec{p} \bar{\sigma}}^{\dagger} c_{\vec{p} \sigma}^{\dagger}\right\rangle c_{\vec{k} \sigma} c_{\vec{q}-\vec{k} \bar{\sigma}}+\text { H.c. }\right], \\
& H^{f 2}(t)=\frac{1}{A} \sum_{\vec{p}, \vec{k}, \vec{q}, \sigma} I(\vec{k}, \vec{p})\left[\delta\left\langle c_{\vec{p} \sigma}^{\dagger} c_{\vec{p}-\vec{q} \bar{\sigma}}\right\rangle c_{\vec{k}-\vec{q} \vec{\sigma}}^{\dagger} c_{\vec{k} \sigma}\right]+J(\vec{k}, \vec{p}) \\
& \times\left[\delta\left\langle c_{\vec{q}-\vec{p} \sigma}^{\dagger} c_{\vec{p} \sigma}^{\dagger}\right\rangle c_{\vec{k} \sigma} c_{\vec{q}-\vec{k} \sigma}+\text { H.c. }\right],
\end{aligned}
$$

where

$$
\begin{gathered}
F(\vec{q})=U-2 V\left(\cos q_{x}+\cos q_{y}\right), \\
G(\vec{k}, \vec{p}, \vec{q})=2 V\left[\cos \left(k_{x}-p_{x}-q_{x}\right)+\cos \left(k_{y}-p_{y}-q_{y}\right)-\cos q_{x}\right. \\
\left.-\cos q_{y}\right], \\
H(\vec{k}, \vec{p})=U / 2-V\left[\cos \left(k_{x}-p_{x}\right)+\cos \left(k_{y}-p_{y}\right)\right], \\
I(\vec{k}, \vec{p})=\left\{-U+2 V\left[\cos \left(k_{x}-p_{x}\right)+\cos \left(k_{y}-p_{y}\right)\right]\right\}, \\
J(\vec{k}, \vec{p})=-V\left[\cos \left(k_{x}-p_{x}\right)+\cos \left(k_{y}-p_{y}\right)\right],
\end{gathered}
$$

and $\bar{\sigma}=-\sigma$. Because the $d$-wave BCS ground state is a spin singlet, its elementary excitations consist of $S=1$ triplet and $S=0$ singlet branches. $H^{f 2}(t)$ captures the $S_{z}= \pm 1$ portions of the triplet fluctuations, studied by Demler et al. ${ }^{8}$ in a different context. $H^{f 1}(t)$ captures singlet and triplet $S_{z}=0$ fluctuations.

Applying linear-response theory to the quasiparticle Hamiltonian $H=H_{\mathrm{MF}}(\vec{P})+H^{\prime}(t)$, we can compute the change in element of density matrix $\delta\left\langle\rho_{a b}\right\rangle$ by using

$$
\delta\left\langle\rho_{a b}(t)\right\rangle=\frac{i}{\hbar} \int_{-\infty}^{\infty} d t^{\prime} \theta\left(t-t^{\prime}\right)\left\langle\left[H^{\prime}\left(t^{\prime}\right), \rho_{a b}(t)\right]\right\rangle_{H_{\mathrm{MF}}},
$$

leading to an equation of the form

$$
(\omega \hat{I}-\hat{M}) \bar{\rho}(\vec{q}, \omega)=H^{\mathrm{ext}},
$$

where $\bar{\rho}$ is a column representing the change in the quasiparticle density matrix. The collective-mode energies $\left\{\omega_{i}(\vec{q})\right\}$ are the eigenvalue ${ }^{20}$ of the matrix $\hat{M}$ which can be read off from 


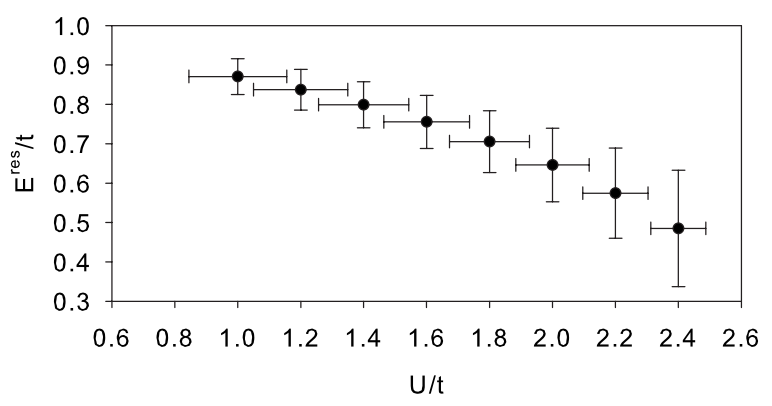

FIG. 2. Resonance-mode energy and weights in spin and pair response functions vs $U$ for $\vec{q}=\vec{Q}$ and doping $x=0.12$. These results were obtained for $\vec{P}=0$ and calculated with $V / t=2.0$ and $t^{\prime} / t=$ -0.3 and $34 \times 34 \vec{k}$ points in the BZ. For each value of $U$, the vertical (horizontal) bar represents the collective-mode weight in the spin (pair) response function. The weights were evaluated using the same response function definitions as that of Tchernyshyov et al. (Ref. 14).

Eq. (14). For a given pairing momentum $\vec{P}$ and excitation momentum $\vec{q}$, the number of particle-hole pairs is proportional to the number of momenta in the Brillouin zone, which we limit by using periodic boundary conditions with a finite quantization area $A=L^{2}$. Diagonalizing $\hat{M}$ is equivalent to performing the boson Bogoliubov transformation and equivalent to summing the ladder and bubble diagrams used to represent the GRPA in diagrammatic perturbation theory.

Typical GRPA extended Hubbard model results for the spin and pair response functions of the $\vec{P}=0$ state at wave vector $\vec{q}=\vec{Q}$ exhibit a single collective mode below the particle-hole continuum with large weight in both responses. In Fig. 2, we illustrate the dependence on $U$ of the energy of this excitation and of its weight in spin-density and tripletpair response functions, with $V, t$, and $t^{\prime}$ (and hence the $d$-wave mean-field state) held fixed. As $U$ increases, promoting antiferromagnetism, the collective-mode excitation energy decreases, its weight in the spin response function increases, and its weight in the pair-excitation spectrum decreases. These properties are consistent with our qualitative effective theory, given the expectation that $K_{Q}^{\mathrm{sp}}$ should decrease with $U$. The opposing variations in triplet-pair and spin-density weights demonstrate that the Berry phase mechanism dominates coupling between antiferromagnetic and pairing phase fluctuations as expected.

\section{DISCUSSION}

In our qualitative discussion we suggested that there should be a strong negative correlation contribution to $\rho$ because the INSR near $\vec{q}=\vec{Q}$ softens with increasing $|\vec{P}|$. Because we perform our calculations with periodic boundary conditions, we estimate the mean-field $\left(\rho^{\mathrm{MF}}\right)$ and correlation ( $\left.\rho^{\text {cor }}\right)$ contributions to $\rho$ by comparing energies at $\vec{P}=0$ and $\vec{P}=\vec{P}^{\text {min }}=(2 \pi / L, 0) ; \rho \approx\left[E\left(\vec{P}^{\text {min }}\right)-E(0)\right] / 2 \pi^{2}$. Figure 3 illustrates the fluctuation-wave-vector dependence of correlation contributions to $\rho$. As expected, we find that ${ }^{22}$ modes near

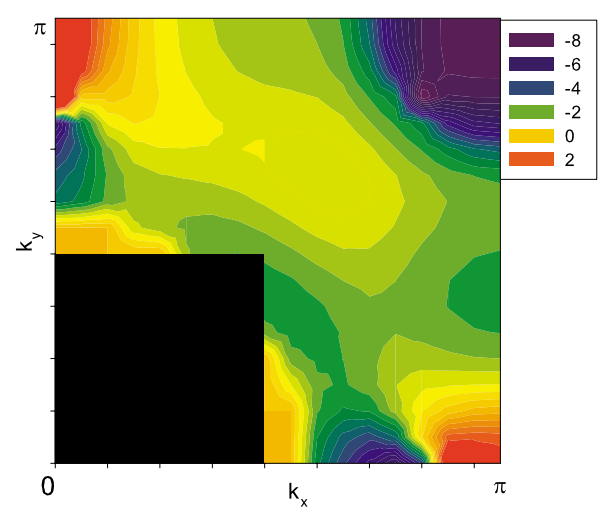

FIG. 3. (Color online) Correlation contribution to $\rho$ from fluctuations with wave vector $\vec{k}\left[\rho^{\operatorname{cor}}(\vec{k})\right.$ for the same model parameters as those in Fig. 1]. $\rho^{\operatorname{cor}}(\vec{k})$ is normalized so that its Brillouin-zone average is $\rho^{\mathrm{cor}} / \rho^{\mathrm{MF}}$, which has the value of -1.6 for these parameters. The GRPA excitation energies in the black area near $\vec{k}$ $=(0,0)$ are imaginary reflecting longer length scale instabilities (Ref. 21) of the extended Hubbard model we use. These longwavelength instabilities are sensitive to model details and independent of the $\rho^{\text {cor }}$ contributions from $\vec{k}=\vec{Q}$.

$\vec{q}=\vec{Q}$ soften, making a negative contribution to $\rho$. This dependence of collective-mode energies on the pair momentum of the superconducting condensate is unusual and is indicative of the microscopic competition between antiferromagnetism and $d$-wave superconductivity. This result contrasts strongly with the absence of any significant dependence of plasmonic collective modes on pair condensate properties in conventional superconductors.

As indicated in Fig. 3, we also find that for the model parameters chosen, collective modes at momenta near $(0,0)$ have complex energies for both values of $\vec{P}$. This finding reflects the tendency of extended Hubbard models, and of real cuprate materials, to longer period density-wave instabilities. ${ }^{21}$ We do not believe that these ubiquitous instabilities, which appear to be material specific, should play an essential role in underdoped cuprate superfluid density suppression since long-wavelength density-wave order will have little impact on near-neighbor antiferromagnetic fluctuations.

We find that $\rho^{\text {cor }} / \rho^{\mathrm{MF}}$ is negative and of order -1 in the underdoped regime when extended-Hubbard-model parameters are in the range thought to represent underdoped cuprates. We conclude that a substantial suppression of the superfluid density due to the pairing-wave-vector dependence of the correlation energy occurs in underdoped cuprates and that it is responsible for the downturn in the critical temperature. Our weak-coupling theory is unable to describe the physics very near the termination of superconductivity on the underdoped side, although there is some indirect evidence from experiment ${ }^{3,4}$ (for example, from the relatively weak temperature dependence of $\rho$ ) that critical fluctuations are important in a relatively narrow doping range. Our explanation for reduced superfluid density in underdoped cuprates is independent of the microscopic origin of the effective nearneighbor interaction responsible for $V$ and hence for $d$-wave superconductivity. 


\section{ACKNOWLEDGMENTS}

This work was supported by the Welch Foundation, the National Science Foundation under Grant Nos. DMR0606489 and DMR-0547875, and the University of Waterloo start-up grant. The authors acknowledge helpful interactions with Dan Arovas, Andrey Chubukov, Eugene Demler, Bernhard Keimer, Patrick A. Lee, M. R. Norman, and Oleg Tchernyshyov. *leewc@mail.utexas.edu

${ }^{1}$ Y. J. Uemura, G. M. Luke, B. J. Sternlieb, J. H. Brewer, J. F. Carolan, W. N. Hardy, R. Kadono, J. R. Kempton, R. F. Kiefl, S. R. Kreitzman, P. Mulhern, T. M. Riseman, D. Ll. Williams, B. X. Yang, S. Uchida, H. Takagi, J. Gopalakrishnan, A. W. Sleight, M. A. Subramanian, C. L. Chien, M. Z. Cieplak, Gang Xiao, V. Y. Lee, B. W. Statt, C. E. Stronach, W. J. Kossler, and X. H. Yu, Phys. Rev. Lett. 62, 2317 (1989).

${ }^{2}$ V. J. Emery and S. A. Kivelson, Nature (London) 374, 434 (1995); J. Orenstein and A. J. Millis, Science 288, 468 (2000).

${ }^{3}$ R. Liang, D. A. Bonn, W. N. Hardy, and D. Broun, Phys. Rev. Lett. 94, 117001 (2005); D. M. Broun, W. A. Huttema, P. J. Turner, S. Ozcan, B. Morgan, Ruixing Liang, W. N. Hardy, and D. A. Bonn, ibid. 99, 237003 (2007).

${ }^{4}$ Iulian Hetel, Thomas R. Lemberger, and Mohit Randeria, Nat. Phys. 3, 700 (2007).

${ }^{5}$ For a recent review, see P. A. Lee, N. Nagaosa, and X.-G. Wen, Rev. Mod. Phys. 78, 17 (2006).

${ }^{6}$ P. W. Anderson, Science 235, 1196 (1987).

${ }^{7}$ In a crystalline superconductor, the Cooper pair momentum $\vec{P}$ is a good quantum number. A state with pairing momentum $\vec{P}$ has a uniform condensate phase gradient $\nabla \phi=\vec{P}$; hence the total energy can be expressed as $E / A=\rho P^{2} / 2$ from Ref. 2 .

${ }^{8}$ E. Demler, H. Kohno, and S. C. Zhang, Phys. Rev. B 58, 5719 (1998), and work cited therein.

${ }^{9}$ H. A. Mook, M. Yethiraj, G. Aeppli, T. E. Mason, and T. Armstrong, Phys. Rev. Lett. 70, 3490 (1993); P. Dai, H. A. Mook, R. D. Hunt, and F. Dogan, Phys. Rev. B 63, 054525 (2001).

${ }^{10}$ H. F. Fong, B. Keimer, D. Reznik, D. L. Milius, and I. A. Aksay, Phys. Rev. B 54, 6708 (1996); H. F. Fong, P. Bourges, Y. Sidis, L. P. Regnault, A. Ivanov, G. D. Gu, N. Koshizuka, and B. Keimer, Nature (London) 398, 588 (1999); H. F. Fong, P. Bourges, Y. Sidis, L. P. Regnault, J. Bossy, A. Ivanov, D. L. Milius, I. A. Aksay, and B. Keimer, Phys. Rev. B 61, 14773 (2000); P. Bourges, Y. Sidis, H. F. Fong, L. P. Regnault, J. Bossy, A. Ivanov, and B. Keimer, Science 288, 1234 (2000).

${ }^{11}$ M. Lavagna and G. Stemmann, Phys. Rev. B 49, 4235 (1994); D. Z. Liu, Y. Zha, and K. Levin, Phys. Rev. Lett. 75, 4130 (1995); N. Bulut and D. J. Scalapino, Phys. Rev. B 53, 5149 (1996).
${ }^{12}$ Ar. Abanov and A. V. Chubukov, Phys. Rev. Lett. 83, 1652 (1999); J. Brinckmann and P. A. Lee, ibid. 82, 2915 (1999); M. R. Norman, Phys. Rev. B 61, 14751 (2000); F. Onufrieva and P. Pfeuty, ibid. 65, 054515 (2002); J. Brinckmann and P. A. Lee, ibid. 65, 014502 (2001).

${ }^{13}$ D. K. Morr and D. Pines, Phys. Rev. Lett. 81, 1086 (1998); S. Sachdev, C. Buragohain, and M. Vojta, Science 286, 2479 (1999).

${ }^{14}$ O. Tchernyshyov, M. R. Norman, and A. V. Chubukov, Phys. Rev. B 63, 144507 (2001).

${ }^{15}$ B. Lake, G. Aeppli, K. N. Clausen, D. F. McMorrow, K. Lefmann, N. E. Hussey, N. Mangkorntong, M. Nohara, H. Takagi, T. E. Mason, and A. Schroder, Science 291, 1759 (2001); E. Demler, S. Sachdev, and Ying Zhang, Phys. Rev. Lett. 87, 067202 (2001); Nicolas Doiron-Leyraud, Cyril Proust, David LeBoeuf, Julien Levallois, Jean-Baptiste Bonnemaison, Ruixing Liang, D. A. Bonn, W. N. Hardy, and Louis Taillefer, Nature (London) 447, 565 (2007); Wei-Qiang Chen, Kai-Yu Yang, T. M. Rice, and F. C. Zhang, Europhys. Lett. 82, 17004 (2008).

${ }^{16}$ N. Nagaosa and S. Heusler, Quantum Field Theory in Condensed Matter Physics (Springer, New York, 1999), Chap. 5.

${ }^{17}$ For a review, see R. Micnas, J. Ranninger, and S. Robaszkiewic, Rev. Mod. Phys. 62, 113 (1990).

${ }^{18}$ For a review, see A. Damascelli, Z. Hussain, and Z. X. Shen, Rev. Mod. Phys. 75, 473 (2003).

${ }^{19}$ D. J. Rowe, Rev. Mod. Phys. 40, 153 (1968); A. H. MacDonald, J. Phys. C 18, 1003 (1985); Y. N. Joglekar and A. H. MacDonald, Phys. Rev. B 64, 155315 (2001).

${ }^{20}$ The eigenvalues of $\hat{M}$ occur in pairs with opposite signs, corresponding to excitation creation and annihilation. The sum in the correlation energy expression is over positive energies only.

${ }^{21}$ J. Zaanen and O. Gunnarsson, Phys. Rev. B 40, 7391 (1989); K. Machida, Physica C 158, 192 (1989); M. Kato, K. Machida, H. Nakanishi, and M. Fujita, J. Phys. Soc. Jpn. 59, 1047 (1990).

${ }^{22}$ Our calculations demonstrate that $C, K^{\mathrm{sp}}$, and $K^{\phi}$ are all weakly frequency dependent and that $K^{\mathrm{sp}}$ decreases because of the reduction in superconducting-state particle-hole excitation energies at finite $\vec{P}$. Wei-Cheng Lee and A. H. MacDonald (unpublished). 